selbe ist hiernach Triphenyläthylammonium, entsprechend der rationellen Formel :

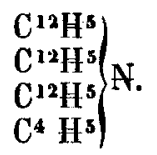

Vom Zimmtsäure-Aldehyd ist es erwiesen, dafs dasselbe unter gewissen oxydirenden Einflüssen leicht in Bittermandelöl und dann in Benzoësäure übergeht, und eben so bekannt ist es, dafs diese beim Erhitzen mit starken Basen Glieder der Phenylgruppe liefert. Denkt man sich Benzoësäure - zwei Kohlenoxydgas, so hat man das Oxyd des Phenylradicals, welches durch einfachen Austausch seines Sauerstoffs gegen den Wasserstoff des Ammoniaks diese Base erzeugen kann. Sieht man von den Nebenerscheinungen ab, so veranschaulicht folgendes Schema auf eine einfache Weise den Vorgang bei der Bildung der Base :

$$
\begin{aligned}
\mathrm{C}^{18} \mathrm{H}^{8} \mathrm{O}^{2}+0 & =\mathrm{C}^{14} \mathrm{H}^{5} \mathrm{O}^{3}+\mathrm{C}^{4} \mathrm{H}^{3} \\
\mathrm{C}^{14} \mathrm{H}^{5} \mathrm{O}^{3}-2 \mathrm{CO} & =\mathrm{C}^{12} \mathrm{H}^{5} \mathrm{O} \\
3\left(\mathrm{C}^{12} \mathrm{H}^{5} \mathrm{O}\right)+\mathrm{NH}^{3} & =\mathrm{C}^{36} \mathrm{H}^{15} \mathrm{H}+3 \dot{\mathrm{H}} .
\end{aligned}
$$

$O b$ es gelingen wird, auf diesem indirecten Wege, vielleicht durch Modification der Temperaturen u. s. w., auch das so viel mir bekannt bis jetzt noch nicht dargestellte $\mathrm{Bi}-$ phenylamin darzustellen, müssen weitere Versuche zeigen.

\title{
Ueber die Wirkung des Chlorzinks auf Hippursäure;
}

\section{von Demselben.}

Die Beobachtung von Mas s on ${ }^{*}$ ), dafs der Alkohol durch Einwirkung von Chlorzink in Wasser und Aether getheilt

*) Compt. rend. VI, 198 und Journ. f. pract. Chem. XIII, 432. 
wird, veranlafste mich, die Wirkung dieses Salzes auf Hippursäure zu untersuchen. Es hat sich daraus ergeben, dals diese Säure hierbei, je nachdem man eine concentrirte Auflösung des Salzes, oder das Salz in fester, wasserfreier Form anwendet, entweder in Benzoësäure und Glycin, oder in Benzonitril verwandelt wird, wie wenn in letzterem Falle das Benzoësäure-Amid, das man in der Hippursäure annehmen könnte, durch Austritt von zwei Atomen Wasser in Benzonitril übergeführt würde, das sich bekannllich nach den Versuchen von Limpricht und v. Uslar*) auch bei der trockenen Destillation der Hippursäure für sich bildet.

Erhitzt man Hippursäure mit einer Zinkchloridlösung, deren Siedepunkt bei 115 bis $120^{\circ} \mathrm{C}$. liegt, so findet schon nach einem halbstündigem Kochen eine allmälige Zerlegung derselben in Benzoësäure und Glycin statt. Durch eine Reihe von Versuchen, bei deren Ausführung ich von den Herren Hipp und Stillesen unterstützt wurde, erwies sich das folgende Verfahren als das zweckmäfsigste. Ein Theil Hippursäure und zwei Theile festes käufliches, in dem gleichen Gewichte Wasser gelöstes Zinkchlorid, oder eine entsprechende Menge einer als Nebenproduct bei der Entwickelung von Wasserstoffgas mittelst Zink und roher Salzsäure erhaltenen neutralen, bei $115^{\circ}$ siedenden Zinkchloridlösung, werden in eine Retorte gefüllt, durch deren Tubus ein Eingufsrohr eingesetzt und die mit einer einfachen, gut abgekïhlten Kolbenvorlage versehen ist. Das Gemisch wird alsdann so lange unter Hinzutropfen des verdampfenden Wassers bei $120^{\circ}$ im Sieden erhalten, bis sich keine Benzoësäure mehr sublimirt. Die Benzoësäure, welche man auf diese Weise erhält, ist selbst bei Anwendung einer stark gefärbten Hippursäure vollständig farblos und rein, und schliefst die Bei-

*) Diese Annalen LXXXVIII, 133. 
mengung der letztern schon aus dem Grunde aus, weil die Hippursäure nicht flüchtig ist und daher im Rückstand bleiben würde, ein Vorzug, den dieses Verfahren vor dem von Dessaignes hat. Erhitzt man Zinkchlorid mit Wasser, so destillirt stets ein kleiner Antheil Salzsäure über und im Rückstand bleibt basisches Zinkchlorid; das käufliche trockene Salz enthält schon diese Beimischung. Dieser Oxydgehalt verursacht nun, dafs stets eine variirende kleine Menge Benzoësäure als benzoësaures Zinkoxyd im Rückstand bleibt; durch Hinzufügung von etwas überschüssiger Chlorwasserstoffsäure und ferneres Erhitzen lälst sich übrigens sehr leicht dieser Theil der Benzoësäure noch gewinnen. Um mich von der Reinheit der auf diese Weise dargestellten Benzoësäure zu überzeugen, habe ich einen Theil des rohen Destillationsproductes direct in Wasser gelöst, filtrirt, krystallisirt und nach dem Abtrocknen in Silbersalz verwandelt. Die hiervon vorgenommenen Analysen stimmten vollkommen mit der Zusammensetzung des benzoësauren Silberoxyds überein.

In der Retorte bleibt, nebst basischem Zinkchlorid, das Glycin; um die Bildung desselben nachzuweisen und dasselbe zu gewinnen, wurde auf folgende Weise verfahren. Der Destillationsrückstand wurde in der vielfachen Menge Wasser aufgelöst, durch Schwefelbaryum das Zink gefällt, mit Schwefelsäure der überschüssige Baryt entfernt und durch Kochen mit Bleioxyd oder besser Bleioxydhydrat die Chlorwasserstoffsäure, so wie die etwa im Ueberschufs zugesetzte Schwefelsäure abgeschieden. Die hierdurch entstandene lösliche Glycin-Bleioxydverbindung wurde dann mit Schwefelwasserstoffgas zersetzt, worauf die vom Schwefelblei abfiltrirte, farblose Flüssigkeit beim Concentriren reines Glycin lieferte. Die Multerlauge verwendete ich zur Darstellung der GlycinKupferoxydverbindung. Um mich von der Identität des auf diese Weise erhaltenen Glycins mit dem auf anderem Wege 
erhaltenen zu überzeugen, führte ich mehrere analytische Bestimmungen aus, und zwar nahm ich neben der Stickstoffbestimmung des reinen Glycins, wie ich es erhalten hatte, die Analysen der daraus dargestellten Kupferoxyd- und Silberoxydverbindung vor. Die Resultate stimmen mit der Formel des Glycins überein, wie sich aus dem Folgenden ergiebt :

0,3973 Grm. Glycin lieferten $1,213 \mathrm{NH}^{4} \mathrm{Gl}+\mathrm{PtGl}^{2}$ $=19,17$ pC. N.

Die Formel $\mathrm{C}^{4} \mathrm{H}^{5} \mathrm{NO}^{4}$ verlangt 18,67 pC. Stickstoff.

Glycinkupferoxyd. - Reines Glycin, oder einfacher ein Theil der erhaltenen Mutterlauge wurde in Wasser aufgenommen, mit der hinreichenden Menge Kupferoxydhydrat kurze Zeit gekocht, von überschüssigem oxyd abfiltrirt und die tief lasurblaue Lösung mit einem gleichen Volumen Alkohol gemischt. Beim Erkalten krystallisirte die Verbindung in tiefblauen, nadelförmigen Aggregaten von bekannten Eigenschaften aus. Die mit dem einmal umkrystallisirten und bei $120^{\circ}$ getrockneten Präparate vorgenommenen Analysen lieferten folgende Zahlen :

I. 0,3441 Grm. lieferten 0,2712 Kohlensäure und 0,1257 Wasser.

II. 0,2560 Grm. lieferten 0,1993 Kohlensäure und 0,099 Wasser.

III. 0,3975 Grm. lieferten 0,141 Kupferoxyd.

\begin{tabular}{lrrrr} 
& berechnet & \multicolumn{1}{c}{ I. } & II. & IlI. \\
$\mathrm{C}^{4}$ & 20,87 & 21,45 & 21,22 & - \\
$\mathrm{H}^{5}$ & 4,34 & 4,06 & 4,30 & - \\
$\mathrm{N}$ & 12,17 & - & - & - \\
$\mathrm{O}^{4}$ & 27,83 & - & - & - \\
$\mathrm{CuO}$ & 34,78 & - & - & $\mathbf{3 5 , 4 7}$.
\end{tabular}


Glycinsilberoxyd. - Frisch gefälltes Silberoxyd wurde mit der Glycinlösung gekocht, filtrirt und im Dunkeln der Krystallisation überlassen. Nach dem Erkalten hatte sich die Verbindung in deutlichen, durchscheinenden Tafeln abgeschieden, die sich im Lichte etwas dunkeler färbten; beim Concentriren schied sich reducirtes Silber ab, während die Glycinverbindung nun in körnigen, warzigen Drusen auskrystallisirt war. Sie zeigte im Uebrigen alle von Boussingaull*) und Horsford ${ }^{* *}$ ) angeführten Eigenschaften. Bei der Analyse wurden folgende Zahlen erhalten :

I. $0,4648 \mathrm{Grm}$. lufttrockener Verbindung lieferten 0,213 Kohlensäure und 0,1111 Wasser.

II. $0,3358 \mathrm{Grm}$. bei 110 bis $120^{\circ}$ getrockneter Verbindung lieferten 0,1678 Kohlensäure und 0,0674 Wasser.

III. $0,2864 \mathrm{Grm}$. bei 110 bis $120^{\circ}$ getrocknet lieferten 0,1393 Kohlensäure und 0,0625 Wasser.

IV. $0,2795 \mathrm{Grm}$. lieferten 0,1655 Silber.

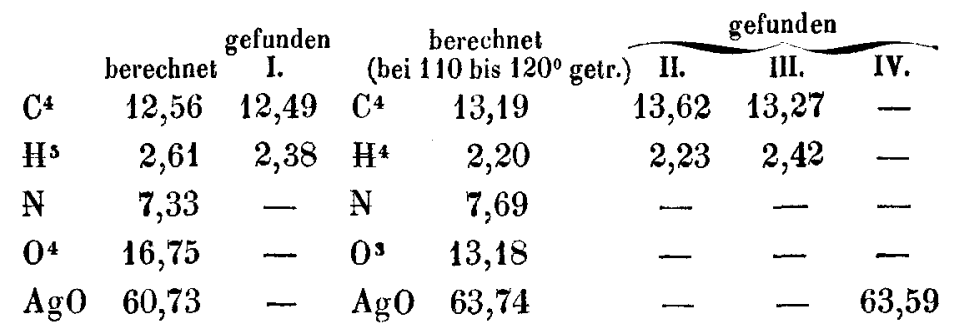

Aendert man das Verfahren beim Behandeln der Hippursäure mit Zinkchlorid in der Weise $a b$, dafs man die trockene Säure mit entwässertem Ziukchlorid gemischt erhitzt, so findet ein dem in dem Vorhergehenden beschriebenen Verfahren in so weit entgegenstehender Vorgang statt, als der Stickstoff in der Hippursäure nicht dem glycinbildenden Paar-

*) Ann. chim. phys. XIII, 113.

**) Diese Annalen LX, 1. 
ling folgt, sondern der Benzoylgruppe, indem sich alsdann vorzugsweise Benzonitril bildet, wie aus folgenden Versuchen zu ersehen ist. Ein Theil Hippursäure wird mit dem gleichen Volumen ausgewaschenen und getrockneten Quarzsand und etwa zwei Theilen festem, möglichst entwässertem Zinkchlorid in einem erwärmten Mörser gemischt, in eine trockene Retorte mit einfacher Kolbenvorlage versehen gefült und rasch auf ungefähr $300^{\circ}$ erhitzt. Die Beimischung von Sand verhindert das Schäumen der Masse und befördert eine gleichmälsigere Temperatur im Innern derselben. War die Feuchtigkeit möglichst ausgeschlossen, so destillirte anfangs nur eine geringe Menge Benzoësäure über, wie es scheint, der in der Masse enthaltenen Feuchtigkeit entsprechend. Ist die angeführte Temperatur einmal erreicht, so destillirt nur Benzonitril über und neben einer geringen Abscheidung von Kohle findet aufserdem eine ruhige Kohlensäureentwickelung stalt. Benzol konnte ich selbst bei wiederholten Versuchen unter den angegebenen Verhältnissen nicht isoliren. Die reichlichste Ausbeute an Benzonitril erhält man dann, wenn man die Temperatur nicht über $350^{\circ}$ hinausgehen läfst; in diesem Falle bleiben alle Nebenerscheinungen, welche von dem einfachen trockenen Destillationsproducte der Hippursäure, wohin besonders Benzol und der eigenthümliche rothe Farbstoff gehören, herrühren, weg. Ich erhielt auf diese Weise 10 bis $12 \mathrm{Grm}$. Benzonitril aus $30 \mathrm{Grm}$. Hippursäure. Abstrahirt man davon, dals das käufliche feste Chlorzink noch Wasser enthält, und dals bei dem Vorbereiten eines Versuches mit einer so hygroscopischen Masse, wie das Chlorzink, ganz unvermeidlich ist, dafs nicht Wasser angezogen wird, durch dessen Gegenwart ein Theil der Hippursäure unter Wasseraufnahme in Glycin und Benzoësäure zunächst zerfällt und so der Ueherführung in Benzonitril entzogen wird, so ist die Menge des erhaltenen Benzonitrils so bedeutend, dafs 
man seine Bildung, wie gesagt, als eine directe Ueberführung aus einem präexistirenden Benzamid betrachten könnte; denn $30 \mathrm{Grm}$. Hippursäure mülsten der Theorie nach gegen 17,5 Grm. Benzonitril bilden. Dafs unter den angegebenen Verhältnissen wirklich stets ein kleiner Theil Hippursäure in Benzoësäure und Glycin zerfällt, geht, neben dem Auftreten der freien Benzoësäure im Destillationsproducte, noch daraus hervor, dafs sich auch in diesem Falle stets eine variirende kleinere Menge von Salmiak, aus zerstörtem Glycin herrührend, im Rückstand findet. Glycin für sich erhitzt färbt sich schon bei 165 bis $170^{\circ}$ gelblich, wird unter Ammoniakentwickelung, Abscheidung von Kohle und Bildung eines weifsen Sublimats, wahrscheinlich kohlensaures Ammoniak, bei $190^{\circ} \mathrm{C}$. vollständig zerstört, und muls demnach unter den angegebenen Verhältnissen Salmiak liefern. In einer concentrirten Flüssigkeit scheint es eine höhere Temperatur vertragen zu können. Aendert man schliefslich die Feuchtigkeitsverhältnisse und Temperaturen ab, so kann man nach Belieben die Endresultate beider näher beschriebenen Verfahrungsarten neben einander beobachten und auf diese Weise bald die einen, bald die andern Producte vorherrschend erzeugen. Also bald mehr Benzoësäure und Glycin (oder Salmiak und Kohlensäure), hald mehr Benzonitril und Kohlensäure u. s. w. Für die Bildung des Benzonitrils ist es immer nöthig, mit wasserfreiem Zinkchlorid auf noch unveränderte Hippursäure einwirken zu können.

Ich habe diese Wirkungsweise des Zinkchlorids auch auf andere, sogenannte gepaarte organische Verbindungen auszudehnen angefangen, und hoffe in Kurzem die Resultate davon mittheilen zu können. 